\title{
Hydrothermal liquefaction of concentrated acid hydrolysis lignin in a bench-scale continuous stirred tank reactor
}

Ivan Kristianto ${ }^{a}$, Susan Olivia Limarta ${ }^{a}$, Young-Kwon Park ${ }^{b}$, Jeong-Myeong Ha ${ }^{a, c}$, Dong Jin Suh ${ }^{a}$, Youngdo Jeong ${ }^{d}$, Jungho Jae , * $^{\text {* }}$

${ }^{a}$ Clean Energy Research Center, Korea Institute of Science and Technology, Seoul 02792, Republic of Korea

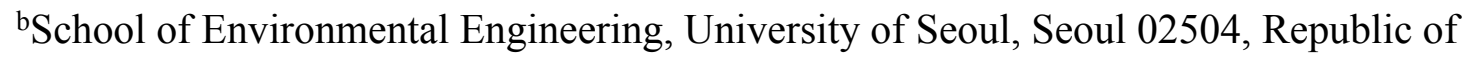
Korea

'Division of Energy and Environment Technology, KIST School, Korea University of Science and Technology, Seoul 02792, Republic of Korea

${ }^{\mathrm{d} C e n t e r}$ for Biomaterials, Korea Institute of Science and Technology, Seoul 02792, Republic of Korea

eSchool of Chemical and Biomolecular Engineering, Pusan National University, Busan 46241, Republic of Korea

*Corresponding author: Tel.: +82-51-510-2989; E-mail: jh.jae@pusan.ac.kr 


\section{Detailed description of the batch reactor experiments}

The depolymerization of CAHL was performed in a 140-ml internal batch autoclave fabricated from Inconel 625, equipped with a four-blade paddle impeller. For a typical experiment, $2 \mathrm{~g}$ of lignin, $20 \mathrm{ml}$ of water, and $25.3 \mathrm{ml}$ of ethanol were loaded into the reactor, giving a water-to-ethanol mass ratio $=50 / 50$ (in the case of hydrogen addition experiments, $2 \mathrm{~g}$ of FA was loaded into the reactor, giving FA-to-lignin mass ratio $=1$ ). The reactor was then sealed, and the air in the headspace of the reactor was purged by nitrogen 2-3 times and pressurized to $1 \mathrm{MPa}$. The reactor was then heated to the desired temperature by using an electric furnace and four cartridge heaters installed on the reactor wall. The stirring rate and reaction time were fixed at $500 \mathrm{rpm}$ and $60 \mathrm{~min}$, respectively. Once the desired reaction time was achieved, heating was stopped, and the reactor was quenched with water. The gaseous products from the reactions were vented out while the liquid/solid mixture was collected by washing the reactor with acetone and then separated by vacuum filtration with pre-weighed Whatman 40 filter paper. After separation, the filter cake was dried at $105{ }^{\circ} \mathrm{C}$ overnight and denoted as solid residue. The filtrate was then evaporated at $70{ }^{\circ} \mathrm{C}$ in reduced pressure using a rotary evaporator, followed by drying at $105{ }^{\circ} \mathrm{C}$ for $3 \mathrm{~h}$ to remove the moisture, and the resulting liquid product was denoted as bio-oil. The product yield was calculated using the following equations.

$$
\begin{gathered}
\text { Yield of bio }- \text { oil }(\%)=\frac{\text { Dry weight of bio }- \text { oil }(\mathrm{g})}{\text { Dry weight of CAHL }(\mathrm{g})} \times 100 \\
\text { Yield of solid residue }(\%)=\frac{\text { Dry weight of solid residue }(\mathrm{g})}{\text { Dry weight of CAHL }(\mathrm{g})} \times 100
\end{gathered}
$$




\title{
Detailed description of the bench-scale reactor setup
}

\author{
Reactor assembly
}

The experimental rig (shown in Fig. 1) comprises an autoclave with internal volume of $700 \mathrm{~mL}$ (reactor), a feeder tank with internal volume of $5 \mathrm{~L}$, a $20 \mathrm{~L}$ of $\mathrm{RO}$ water reservoir, a 10L of acetone reservoir, 2 HPLC pumps, 2 filters, 1 heat exchanger (using tap water as a coolant), 2 ball valves, 10 needle valves, and a back pressure regulator (BPR) valve. Two filters were installed on the reactor system whereas one is used as a backup in case of clogging occurred during the reaction. The feeder tank, reactor, and filters were ensured to be securely sealed and the feeder was then pressurized into 210 barg (BV 1 and NV 1 were in close position while NV 10 was in open position). The mixture of lignin and solvent was isolated once they were loaded into the feeder tank (NV 10 and BV 1 are in close position).

\section{Leak test and reactor heating}

Filter 1 acted as the main filter while filter 2 as a backup filter. Their corresponding valves are therefore adjusted into open position before the water was fed into the reactor system (NV2, NV8, BV2 were in open position; NV $3-7$ and NV 9 were in close position). The reactor line was then flushed by RO water to ensure no contaminants resided on the reactor line (NV1, BV2 are in open position) for approximately $30 \mathrm{~min}$. The flow rate was set to the a specific value corresponding to the desired space time and the whole system was then pressurized into 200 barg by adjusting the opening of the BPR and ensured the pressure of the system was stable for $3 \mathrm{~h}$. Once no leak was 
observed on the reactor lines (including the valve fittings), the jacket furnace was then securely attached, cartridge heaters were inserted into the reactor's wall, and the thermocouples were connected to the control panel. The set point temperatures of furnace and cartridge heaters were then adjusted so that the desired reaction temperature was achieved. It took approximately $150 \mathrm{~min}$ and $210 \mathrm{~min}$ to achieve $300{ }^{\circ} \mathrm{C}$ and $350{ }^{\circ} \mathrm{C}$. During the reactor heating, water was pumped continuously through the reactor system (NV 10 and BV 1 are in close position), NV1, BV2 are in open position).

\section{Depolymerization of CAHL}

Once the pressure and the temperature were steady, pump 1 was stopped, NV 1 was shut off, and NV 10 was adjusted into open position, and the pump was started again. BV 1 was adjusted to fully open right after the pump worked. At this situation, the mixture of lignin and solvent was fed into the reactor by pressure difference, the reaction was initialized. Plastic beaker was used to collect the product mixture. For safety reason, the pump was set so that pressure of the system will not exceed 250 barg and the pressure and temperature of the system were monitored during the operation.

\section{Post reaction and backwashing}

The feeding was stopped after $5 \mathrm{~h}$ operating by shutting off BV 1 and NV 10, and adjusted NV 1 to fully open. At this point, water was delivered through the line to purge out the reaction mixture that still resided on the reactor's line and the outlet stream was still collected in a plastic beaker. After the line system was purged for $2 \mathrm{~h}$, the heaters 
were shut down, and the reactor was cooled down to ambient temperature. The reactor system was then depressurized to atmospheric and the remaining product mixture on the filter was collected by opening NV 4. For backwashing by acetone, NV 2 and NV 8 were adjusted to fully close while NV 6 and NV 4 were fully open. The liquid from backwashing was collected in a different beaker until the colour was transparent, indicating the remaining bio-oil was washed away from the filter line. 
Comparison of the physicochemical properties of CAHL and Kraft lignin (KL)

Table S1. Elemental and proximate analysis results of lignin samples.

\begin{tabular}{|c|c|c|c|c|c|c|c|c|c|c|c|}
\hline \multirow{2}{*}{ Feedstock } & \multicolumn{7}{|c|}{ Elemental analysis (wt\%) } & \multicolumn{4}{|c|}{ Proximate analysis (wt $\%)$} \\
\hline & $\mathrm{C}$ & $\mathrm{H}$ & $\mathrm{N}$ & $\mathrm{S}$ & $\mathrm{O}$ & $\mathrm{O} / \mathrm{C}$ & $\mathrm{H} / \mathrm{C}$ & Moisture & $\begin{array}{c}\text { Volatile } \\
\text { matter }\end{array}$ & $\begin{array}{l}\text { Fixed } \\
\text { carbon }\end{array}$ & Ash \\
\hline CAHL & 53.0 & 5.2 & 1.3 & 1.2 & 39.3 & 0.56 & 1.17 & 3.6 & 56.5 & 31.0 & 8.9 \\
\hline KL & 61.9 & 5.7 & 0.7 & 0 & 31.7 & 0.38 & 1.11 & 3.4 & 54.3 & 38.5 & 3.8 \\
\hline
\end{tabular}

\section{GPC chromatograms of bio-oils at various reaction conditions}

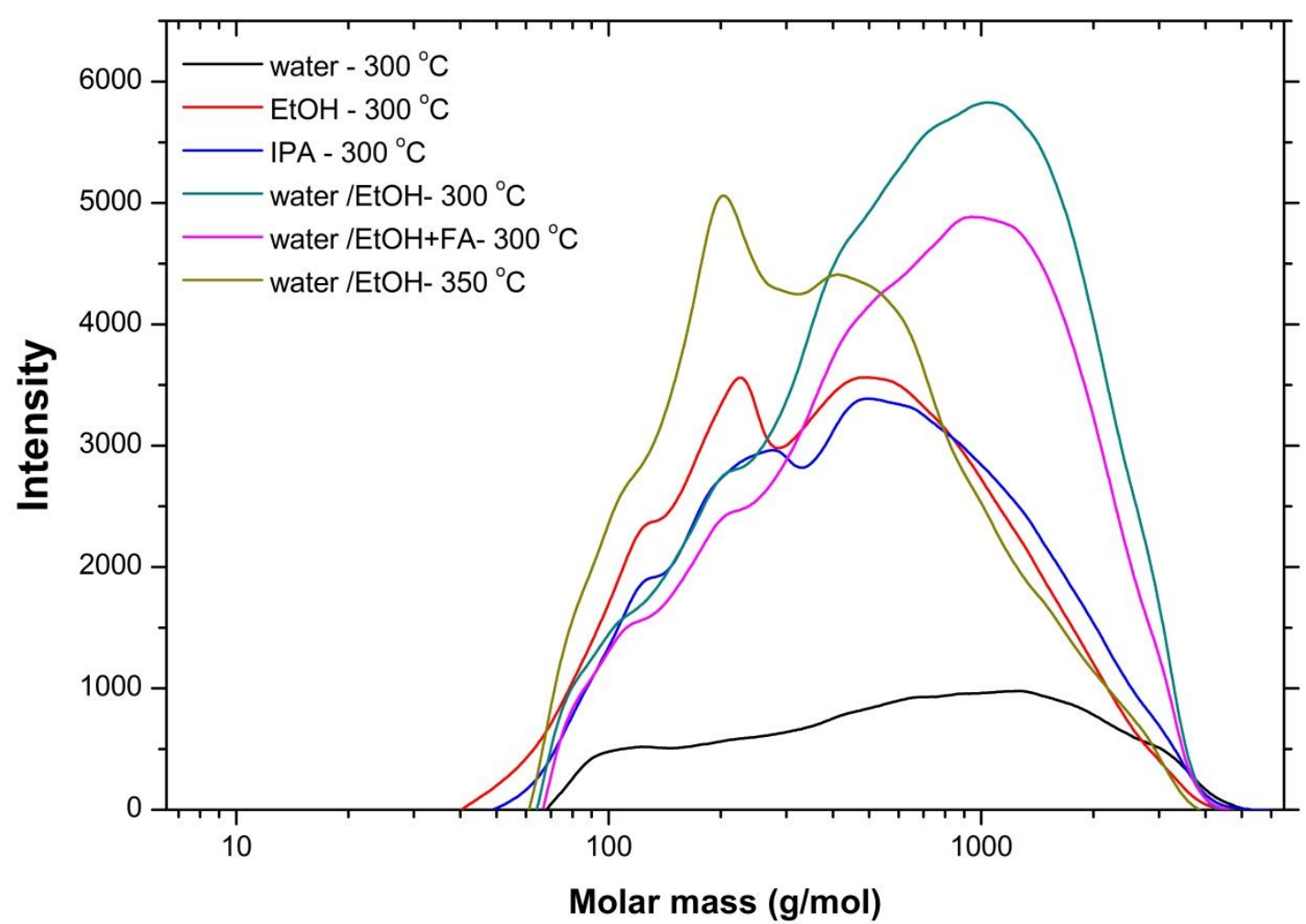

Figure S1. GPC chromatogram of bio-oils obtained from batch experiment: Effect of co-solvent and formic acid addition 

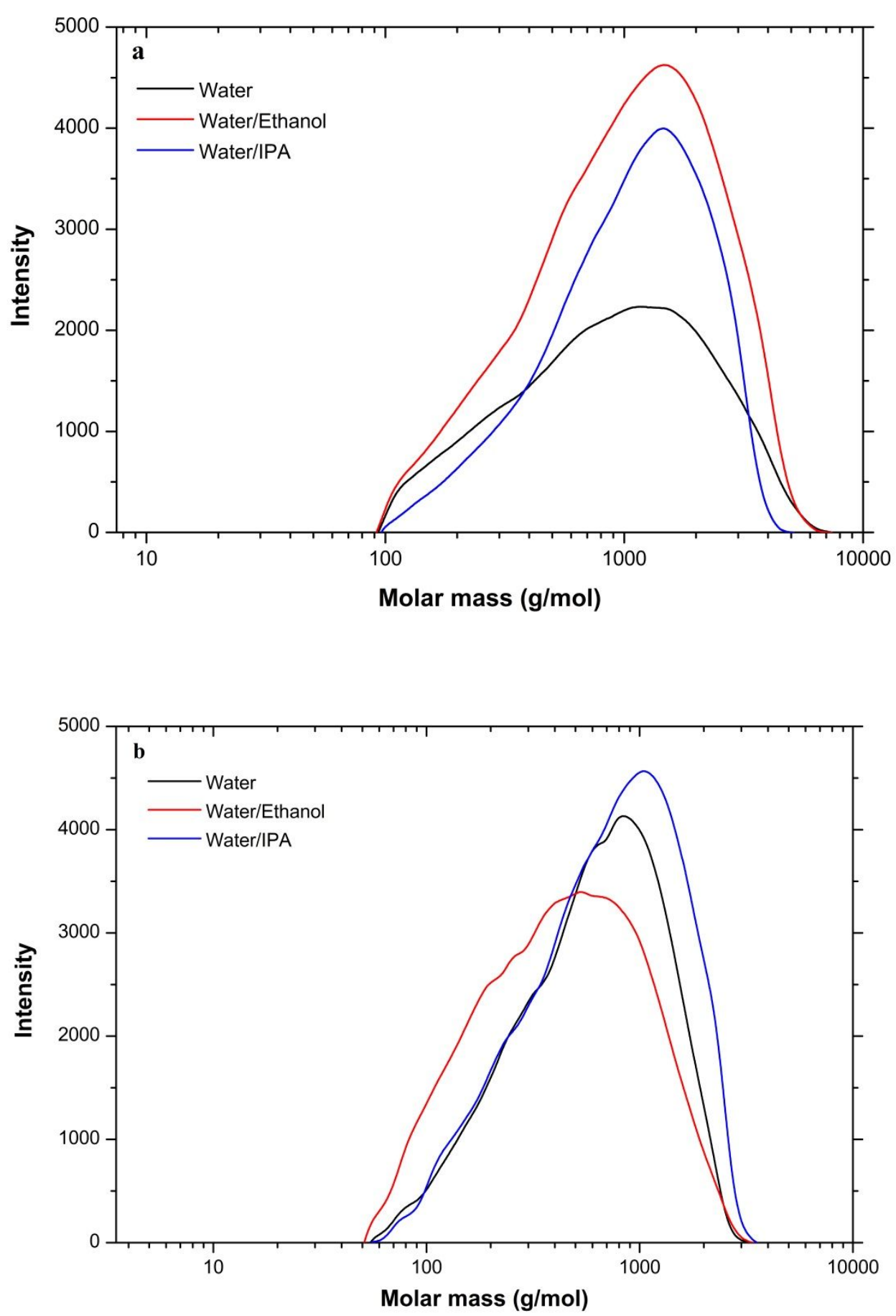

Figure S2. GPC chromatogram of bio-oils with different solvents at (a) $300{ }^{\circ} \mathrm{C}$ and (b) $350{ }^{\circ} \mathrm{C}$, respectively. 


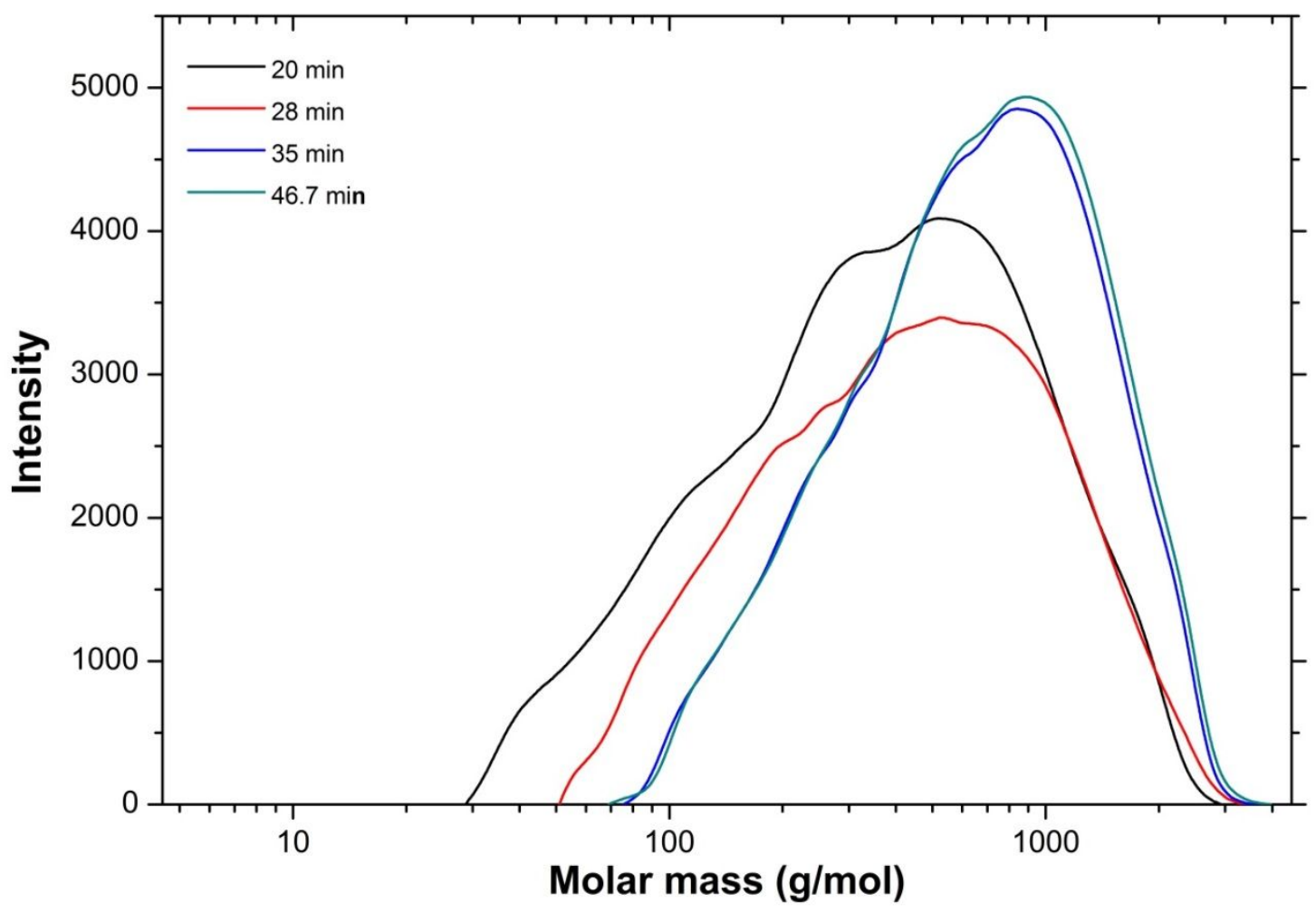

Figure S3. GPC chromatogram of bio-oils at different space times. Reaction condition: reaction temperature of $350^{\circ} \mathrm{C}$ and water/ethanol co-solvent. 